\title{
The transient behaviour of an input protection.
}

Kees de Kort (1), Jan Marc Luchies (2), and Joris J. Vrehen (1)

1 Philips Research Laboratories Eindhoven

Prof. Holstlaan 4, WAY-31, 5656 AA Eindhoven, The Netherlands

2 MESA Research Institute, University of Twente

PO 217, 7500 AE Enschede, The Netherlands

\begin{abstract}
The high frequency behaviour of input protections has been measured with electro-optic sampling. These measurements allow the determination of the time dependence of the voltages at internal nodes as well as the time dependence of the current through the input protection. Simulations are performed using a detailed model of the input protection and a simplified model of the integrated circuit, including connections. The measurements are vital to the development of the model, which ultimately aims at explaining the differences found in Human Body Model testing and Charged Device Model testing.
\end{abstract}

\section{Introduction}

When an electrically charged person touches an object at a different potential a discharge may occur. This phenomenon of electrostatic discharge (ESD) can also occur in the handling of integrated circuits (IC's), giving rise to voltage pulses at one or more pins connecting the IC to the outside world. Therefore, each pin of the IC is usually protected by a so called ESD protection circuit. The function of the protection circuit is twofold: firstly, it clamps the voltage to a sufficiently low level that can be sustained by the IC and secondly, it creates a current path that can handle the power present in the ESD pulse. These functions are commonly performed by specially designed diodes or transistors. The performance of the protection circuit is determined by standardized ways of testing. The human body model (HBM) is a well known standard [1], and several others, like the machine Model (MM) and the charged device model (CDM) [2], are under discussion. For test purposes, ESD pulses are generated by charging a capacitor to a specified voltage and a subsequent discharge by connecting it to the pin of a device under test (DUT). The standard(s) prescribe the value of the capacitor and the impedances of the connection to the pin of the DUT. For example, the HBM uses a capacitor of $100 \mathrm{pF}$ which is discharged through a resistance of $1500 \Omega$ and an induction of $20 \mu \mathrm{H}$ in series with the DUT. The MM proposes a much smaller resistance and a different value of the inductance, whereas 
the CDM proposes to charge the device first and then to discharge it through a specified impedance network.

The voltage waveforms appearing at the pin of the DUT depend on the applied test standard as well as on the characteristics of the protection and the interconnections within the DIT. The detailed shape of these waveforms is not known, but in going from HBM to CDM they become more stecp. Therefore, the response of the protection depends on the way of testing and it can occur that a given implementation of a protection meets the criteria for HBM testing, but fails the criteria for CDM testing. These criteria are commonly expressed as the voltage used to generate the most energetic input pulse which can be sustained by the DUT. For a better understanding of the behaviour of the protection circuitry, knowledge of the actual waveforms at the internal nodes is needed [5]. Although the current pulse which enters the circuit can be adequately measured, the measurement of short voltage pulses with relatively high amplitude, existing at an internal node, is not an every day affair.

Electro-optic (e-o) sampling $[6,7]$ is one of the few techniques that meets the demands expressed above. Since this method presents a very low load to the circuit under investigation, it has a minor influence on waveforms. Therefore, in this work e-o sampling will be used to measure the internal waveforms. Being a sampling technique, it requires signals with much higher repetition rates than possible with the standard test methods. This raises the question if findings for experiments with high repetition rates have any meaning for the behaviour at low repetition rates. It is one of our aims to show that the time dependence and amplitude of waveforms with high repetition rate provides useful insight, even for single pulses. Here, it must be emphasized that in this study the amplitude of the current pulses is kept low enough to prevent damage, either to the input protection or to the remaining circuitry. The prime interest is to study the transient behaviour of the protection circuitry, under the condition that no degradation is taking place. The experimental set-up used in these experiments is described in section 2 .

In order to simulate the behaviour of the input protection a model is developed which accounts for the behaviour of the transistor(s) in the input protection at high currents and high frequencies. This is described in section 3. To simulate voltages at internal nodes, not only the input protection, but also the input and output connections as well as a simplified model for the impedances within the IC have to be included. Simulations obtained with this model are compared with experiments and these results are displayed and riscrussed in section 4.

\section{$2 \quad$ Experimental set-up}

The experimental set-up shown in fig. 1 is basically the same as described in [8]. The $\mathrm{Bi}_{12}, \mathrm{SiO}_{20}$ e-o crystal is equipped with a needle and a reference electrode. By using feedback to the reference electrode waveforms can be measured with calibrated amplitude, giving an accuracy of around $4 \%$ for waveforms with an amplitude above $1 \mathrm{~V}$. The noise corresponds to $2 \mathrm{mV} / \sqrt{\mathrm{Hz}}$, but for good reproducibility the needle on the e-o probe has to make good contact with the node of interest.

A frequency generator is used to trigger a pulse generator, the output of which is fed through a coaxial cable to the pin of the DUT. Unloaded, the amplitude of the pulse 


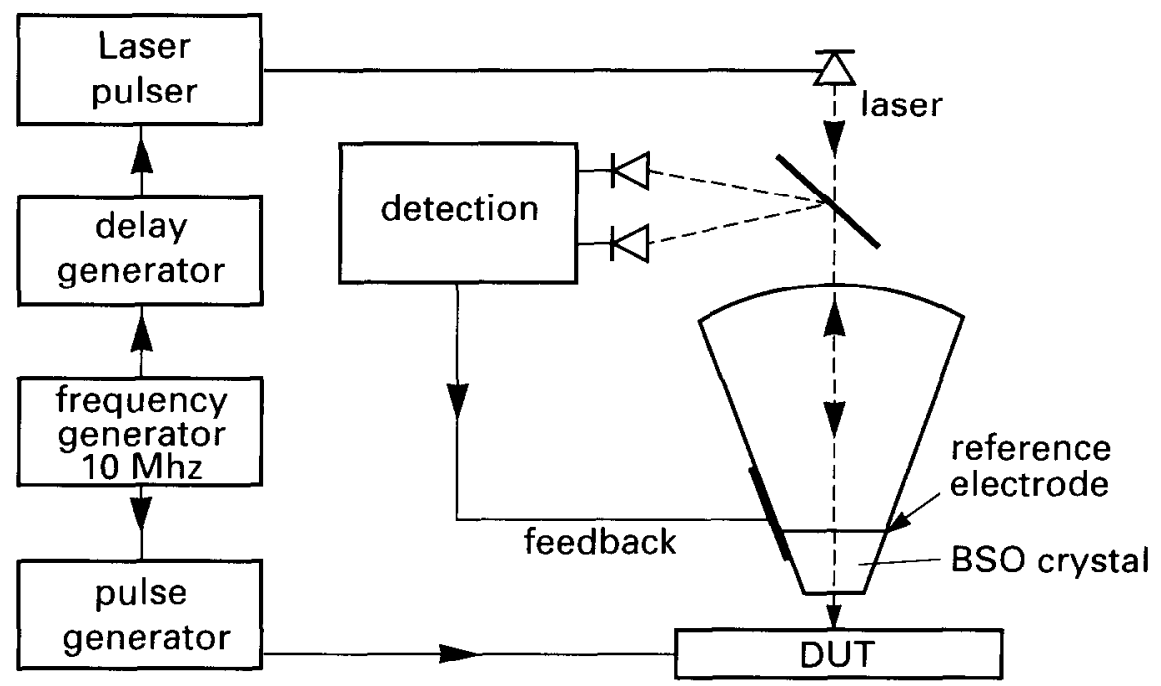

Figure 1: Set-up for the measurements of ESD waveforms with e-o sampling.

generator can be varied between $0 \mathrm{~V}$ and $32 \mathrm{~V}$. The duration of the voltage pulses as well as their rise and fall times can be varied, but in this paper we will limit ourselves to input pulses of fixed shape and amplitude. A second output of the frequency generator is used to trigger a diode laser, which emits optical pulses of $50 \mathrm{ps}$ duration. The delay generator is used to 'scan' the optical pulses over the voltage pulse. The optical pulses are directed into the $\mathrm{Bi}_{12} \mathrm{SiO}_{20}$ crystal where they 'sense' the electric field within the $\mathrm{Bi}_{12} \mathrm{SiO}_{20}$ and upon reflection the detection optics converts the optical signal into an electrical signal, which is proportional to the voltage drop over the e-o crystal. With feedback on, the detected signal is kept at zero and then the signal amplitude at the reference electrode equals the signal amplitude on the circuit node at the time of sampling.

The lay-out of the IC used in these experiments is schematically depicted in fig. 2a. The

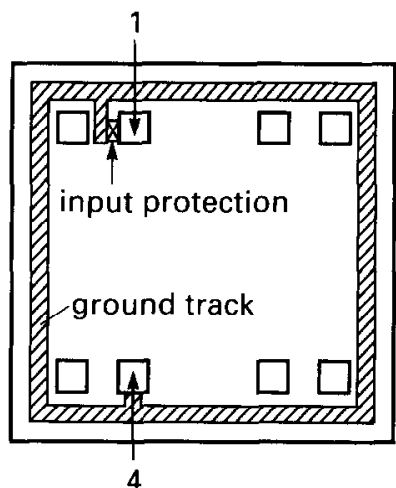

a)

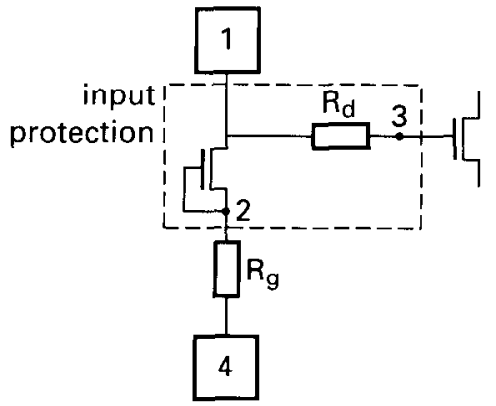

b)

Figure 2: a) Lay-out of IC with input protection, b) electrical scheme including the input protection and definition of circuit nodes. 
input protection to be studied is situated between bondpad 1 and a metal line connected to bondpad 4. With a focused ion beam (FIB) small probe pads are made to make nodes 2 and 3 accessible. This is achieved by making a hole in the scratch protection followed by filling it with tungsten. The same procedure is used to make small probe pads on the metal lines directly connected to bondpads 1 and 4 , which allows to control the quality of measurements made on FIB-grown probe pads. The IC is mounted in a DIL package. The inner conductor of the coaxial cable coming from the pulse generator is connected to a pin bonded to bondpad 1, while the shield of the coaxial cable is connected to the pin bonded to bondpad 4 . Internal measurements are performed at both bondpads and on internal nodes 2 and 3 as shown in fig. $2 \mathrm{~b}$.

\section{Simulations}

To support and further understand the response of the protection circuit on large sig. nal transients, circuit simulations were carried out using the circuit simulator Pstar [9]. Herewith, we hope to predict the behaviour of protection circuits under ESD conditions. Differences between Human Body Model and Charged Device Model, implying different pulse rise times, can then be simulated.

The equivalent circuit model of the experimental set-up is shown in fig. 3 .

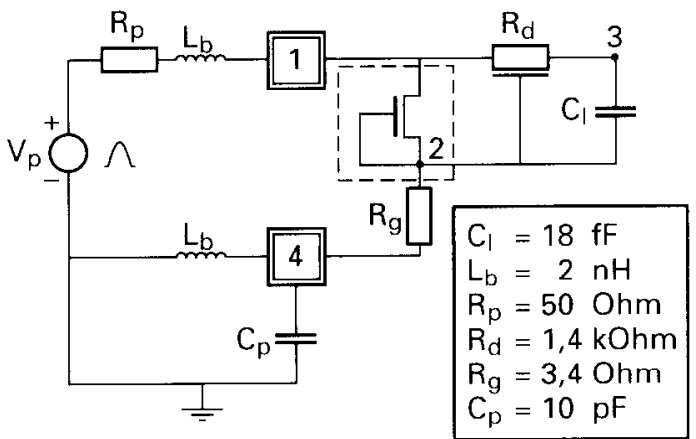

Figure 3: Electrical scheme as used in the simulations, including pulse generator, external connections, internal connections and input protection.

The pulse generator $\mathrm{V}_{\mathrm{p}}$ and its internal resistor $R_{p}$ together with the bondwires $L_{b}$ and some parasilic capacitances form the external circuit. The protection circuit is built up from an 100/1.2 NMOS transistor, the polysilicon resistor $R_{d}$ and the input gate modelled with $C_{l} . R_{g}$ is the resistance of aluminum interconnection between the input protection and bondpad 4 .

To describe the input protection a bipolar electrical transistor model will be used, as illustrated in fig. 4, which is basically an Ebers-Moll model with a number of extensions describing mechanisms playing an important role in breakdown.

For evaluating the main mechanisms involved in breakdown of a parasitic bipolar transistor, a coupled electrical-thermal transistor model is used. The electrical model is based on the one used by Latif and Bryant [10], which includes avalanche and high injection.

Avalanche is often considered to be the initiating mechanism for snap-back, which has been included in the lateral transistor model through the Miller formula. High injection 
in the base is included by modelling the transition in the voltage-current relationship. An empirical relation describing the current dependency of the current gain is also included. The collector resistance $R_{c}$ is an equivalent spreading resistance of the drain. The base resistance is formed by the substrate resistance. Additionally, the depletion capacitances of the base-emitter, base-collector junctions as well as the diffusion capacitance are added to the model, to simulate large signal transients.

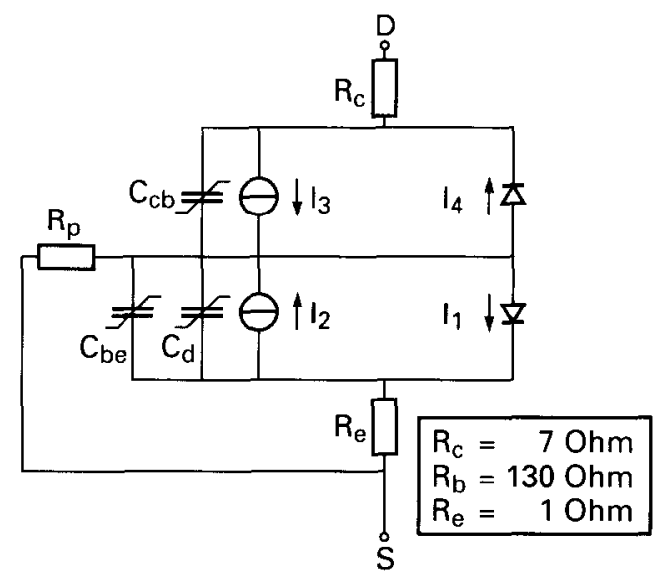

Figure 4: Model of input protection transistor. The arrows indicate the direction of currents il ...i4.

The electrical model is coupled with a model describing the thermal behaviour of the IC by means of an electrical analogue. Coupling between the electrical and thermal model takes place via power dissipation in the protection transistor. A number of model parameters were derived from measurements on single devices, the other model parameters were derived from $2 \mathrm{D}$ device simulations. The simulated waveforms are depicted in fig. 5 and they will be discussed in the next section.

\section{Results and Discussion}

The measurements in fig. 5 are performed for an electrical input pulse with a half width of $8 \mathrm{~ns}$ and $4 \mathrm{~ns}$ rise and fall times. This waveform has an amplitude of $23.8 \mathrm{~V}$. In fig. $5 \mathrm{a}$, the continuous and dotted curves are measured at bondpad 1 and node 3 . It is seen that the amplitude is clamped at approxinately $12 \mathrm{~V}$ and before reaching this level the waveform shows a small peak, which signals the onset of snap-back in the input protection. The simulated countcrpart of fig. $5 \mathrm{a}$ is shown in fig. $5 \mathrm{~b}$. The resistances and voltage dependent capacitors in the transistor model (see fig. 4) are determined from transistor measurements and their values are not varied in the simulations. The protection transistor goes into snap-back when the input voltage becomes slightly higher than the first breakdown voltage at $14 \mathrm{~V}$. Hereafter, the amplitude is clamped at approximately $12 \mathrm{~V}$, which is in agreement with the measurements. It turns out that the appearance of a small peak in the simulations is critically dependent on the way the model numerically treats the onset of snap-back. 
MEASUREMENTS

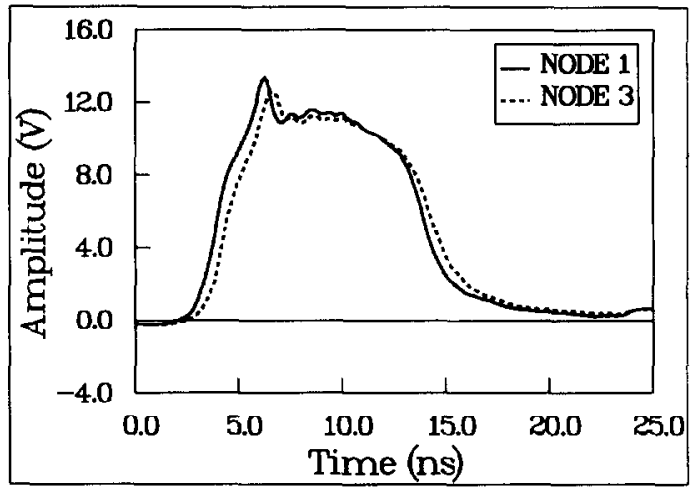

a)

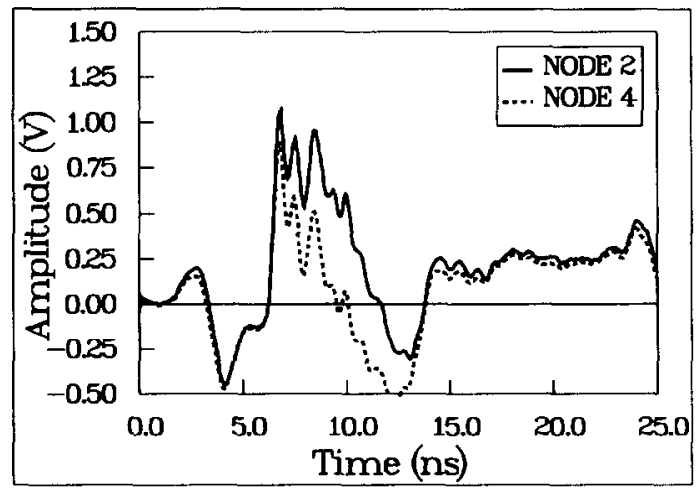

c)

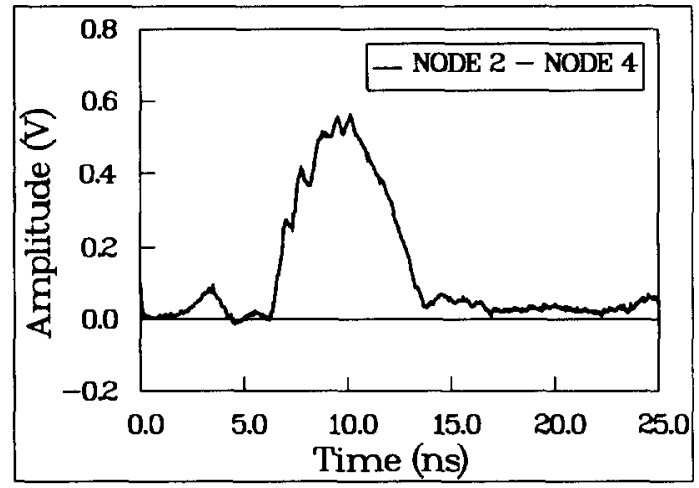

e)
SIMULATIONS

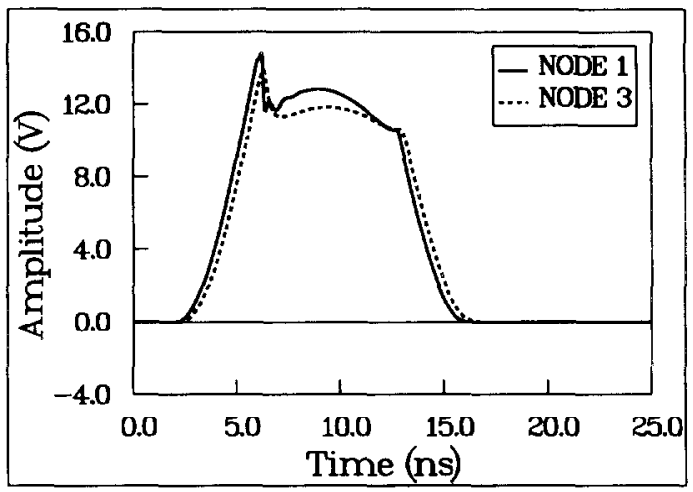

b)

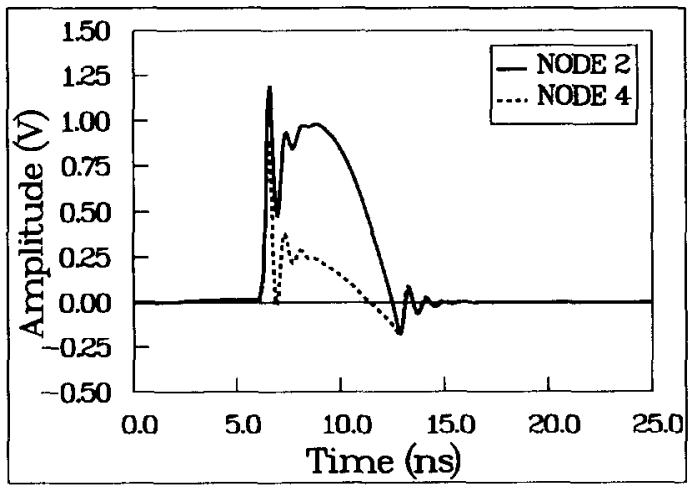

d)

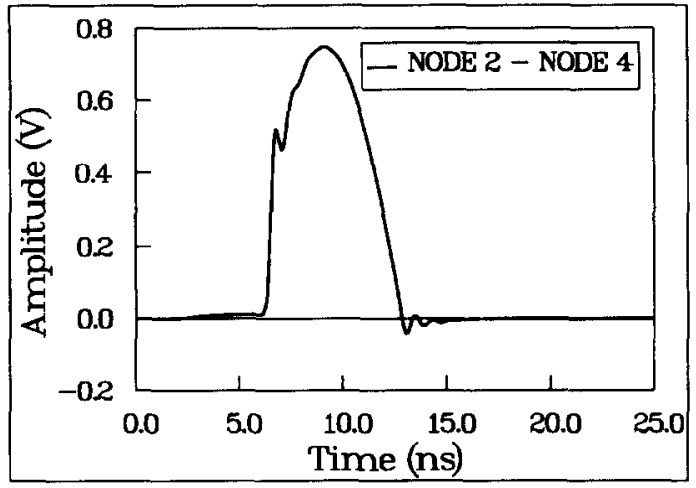

f)

Figure 5: Measured and simulated waveforms

a,b) measurement and simulation at node 1 and 3

c,d) measurement and simulation at node 2 and 4

$\mathrm{e}, \mathrm{f})$ measurement and simulation of the difference between node 2 and node 4

Since the simulation does not include time effects (other than those caused by passive elements), the falling slope of the small peak is almost instantenous, whereas the 
measurements show that it last about $0.5 \mathrm{~ns}$. Therefore, improvements on the switching behaviour of the model are needed. When the protecton transistor is switched off, a little peak appears, which is not present in the measurements. This is probably due to inaccurate modelling of charge carriers in the base.

The small shift between the waveforms at bondpad 1 and node 3 is caused by the delay arising from the resistance $R_{d}$ and the distributed capacitance of this resistor together with $C_{l}$ (see also fig. 3). From the process parameters the sum of both capacitors is estimated to be $68 \mathrm{fF}$. Together with the measured load of the e-o tip (150 fF) and the value for $R_{d}$ of $1.4 \mathrm{k} \Omega$ we arrive at a delay of $300 \mathrm{ps}$ which is in reasonable agreement with the measured shift of $400 \mathrm{ps}$. It is clear that for even faster input transients (as can be expected for CDM tests) this RC time not only shifts, but also reduces the amplitude of the transient present at the first gate connected to the input protection. Also, the capacitance of the e-o probe should be reduced below $10 \mathrm{fF}$ to prevent interference for measurements on points like node 3 .

In fig. $5 c$ measured waveforms at internal node 2 and bondpad 4 are shown. Immediately apparent is the oscillatory behaviour in both waveforms. Since both waveforms are in phase it is concluded that the impedance between node 2 and bondpad 4 is largely resistive. The oscillatory behaviour is caused by the impedances making up the connections to the IC (bondwires, package) and the capacitance of the input protection, rather than circuitry within the IC. By varying the inductance $L_{b}$ and the capacitor between bondpad 4 and ground, the simulated oscillation period, shown in fig. $5 \mathrm{~d}$, could be brought in agreement with the measurements. In this way we arrived at values of $2 \mathrm{nH}$ for $L_{b}$ and 10 $\mathrm{pF}$ for the capacitance. The amplitude of both simulated and measured waveforms is in good agreement during the input pulse, except for the period before snap-back, where the measurements show a much more distinct feature in the voltage than is observed in the simulations. After snap-back, the substrate voltage depends on the position within the IC. Close to the input pad it can be as high as $1 \mathrm{~V}$ and at the same time it is only $0.5 \mathrm{~V}$ in the neighbourhood of bondpad 4. Therefore, voltages accross the gate oxide of transistors within the IC vary, even if they are connected to the same input. It is expected that these effects become even more pronounced when faster transients are applied to the IC. Also, impedances within the IC should be modelled in more detail in order to determine which transistor in the IC experiences the largest voltage transient across its gate oxide and is therefore most prone to gate oxide damage.

The almost constant voltage level, observed after the input transient has finished, is completely absent in the simulations. The reason for this discrepancy is not understood. Further disagreements in some of the details are not surprising, since we have made large simplifications in the impedance network formed by the IC and its interconnections. It is clear that more refinements are necessary to bring about a detailed agreement.

From the foregoing discussion it is derived that the current is proportional to the difference of the signals at node 2 and bondpad 4 , this difference being displayed in fig. $5 \mathrm{e}$ and 5f for respectively the measurement and the simulation. The agreement between measurement and simulation is quite satisfactory, be it that the simulations show a somewhat larger amplitude. The amplitude of the difference signal is determined by the product of current and resistance, both of which are determined by independent measurements. However, the resistance is determined by a DC measurement whereas the measurements 
reveal frequency components well above $1 \mathrm{GHz}$, which could account for a small variation in the actual resistance between node 2 and bondpad 4 . Alternatively, some of the current could flow through the substrate, also giving rise to a lower resistance.

\section{Conclusions}

There is a reasonable agreement between the measured and simulated response of an ESI protection to a transient with an amplitude, high enough to switch on the protection transistor, but low enough to prevent damage to the $\mathrm{IC}$ or the protection itself. The development of a simplified model is guided by a dual approach: simulations and measurements. It is this very twinning which provides the directions in which the model has to be improved. In this way, it was found that model improvements have to be focused on the features occuring around snap-back. The importance of the impedances formed by the interconnections to and within the I $('$ is made clear and actual values for these impedances could be arrived at. Partly, these impedances determine the difference between HBM and CDM testing. An example is the difference in voltage transients appearing across the gate oxide of transistors in the IC. It is anticipated that the final model will help to improve our understanding of these differences more fully.

\section{Acknowledgements}

It is a pleasure to thank Fred Kuper for many fruitful and helpful discussions on the subject of ESD protections. Without his stimulation and kindly supplied samples this project would not have becn possible.

\section{References}

[1] MIL-STD-883C, Method 3015.3, 'Flectrostatic Discharge Sensitivity Classification', November $29,1985$.

[2] L. Avery, Proc. 9th EOS/ESD Symposium, (1987), 88.

[3] C. Duvvury, R.N. Rountree, and O. Adams, 26th annual proceedings reliability physics, (1988), 19.

[4] Ajith Amerasekera, Jan Verweij, Quality and Relibility Engineering International, Vol. 8., (1992), 259.

[5] Y. Fong and C. Hu, 27th annual proceedings reliability physics, (1989), 77.

[6] J.A. Valdmanis,G. Mourou and C.W. Gabel, Appl. Phys. Lett. 41 (3) (1982), 211.

[7] J. Nees, G. Mourou, Electronic Letters 22 (17) (1986), 918.

[8] Kees de Kort and Joris J. Vrehen, Microelectronic Engineering 16, (1992), 341.

[9] Pstar, Philips Electronic Design \& Tools (ED\&T), 1993.

[10] M. Latif and R. Bryant, IEEE Journal of Solid State Circuits, vol SC-16, (1981), 8. 